Mohammad Atif Faiz Afzal ${ }^{1}$

${ }^{1}$ Affiliation not available

June 12,2020

\begin{abstract}
Please use this section to briefly summarize the manuscript, put its findings into the context of the existing state-of-the-art, indicate the overall significance of the work, provide an impression of the overall quality of the work and its strengths, state whether there are any major flaws or weaknesses, and recommend a course of action (Accept, Revise, Reject) to the editor.

Please be aware that that IJQC's Executable 8 Interactive Article Special Issue feature a Transparent Peer Reviewer workflow, which means that the reviewer reports associated to the article published (like this one) will be published alongside the accepted articles. Let us know here if your name can be publicly associated to your report once published or should anonymized. Please note that before acceptance of the article, your identity will be unknown to the authors (The referee report in Authorea shows your name but this is visible only to the editor).
\end{abstract}

Click on "Submit Review" above the title to send your assessment to the editor

\title{
1 Referee Report
}

The manuscript at hand reports an overview of the computational alchemy approach to evaluate catalysts and provides a standard procedure to perform such computations for specific applications. The paper does not present any new research, but rather outlines the computational alchemy method and provides an opensource and user-friendly tool to the community for them to efficiently perform their own catalyst search. Computational alchemy is an approximate method that allows us to obtain large data from a single DFT calculation, thus allowing us to perform large-scale screening of catalysts. This method was initially described by Straatsma and McCammon in 1992 and has been recently shown to hold great promise in accelerating catalyst search with less computational effort. The current work gives a detailed review of this approach and describes all the approximations that go into the method

The manuscript provides two examples of implementation of the method, which include prediction of binding energies for $\mathrm{OH}$ adsorption on $\mathrm{Pt}(111)$ surface and predictions of reaction barriers of $\mathrm{CH} 4$ * dehydrogenation on $\mathrm{Pt}(111)$ surface. These two examples serve as benchmarks of the method for binding energy estimation and reaction barrier estimation, respectively. Additionally, the authors have developed a web interface, using Jupyter Notebooks, for an easy understanding of the implementation of the method.

In conclusion, the manuscript is predominantly well written, presents a clear description of the methodology, benchmarks the methods using two examples, and provides clear and user-friendly software. I believe the tools provided will be of significant use to the materials design community and aid in the design of new catalyst materials. My recommendation, therefore, is to accept the manuscript to be published in the International Journal of Quantum Chemistry.

I identified a few typos in the manuscript, which I mentioned as comments on the Authorea platform. 
